\title{
Chronic Pain in Hospitalized Infants: Health Professionals' Perspectives
}

\author{
Rebecca R. Pillai Riddell, ${ }^{*}{ }^{\dagger}$ Bonnie J. Stevens, ${ }^{\ddagger}$, Patricia McKeever, ${ }^{\ddagger}$, , t† Sharyn Gibbins, ${ }^{\S, \|}$ \\ Liz Asztalos, "I. Joel Katz, *,** Sara Ahola, * and Laila Din* \\ * Department of Psychology, York University, Toronto, Ontario, Canada. \\ ${ }^{\dagger}$ Department of Psychiatry Research, Hospital for Sick Children, Toronto, Ontario, Canada. \\ ${ }^{\ddagger}$ Lawrence S. Bloomberg Faculty of Nursing, University of Toronto, Ontario, Canada. \\ ${ }^{\S}$ Child Health Evaluative Sciences Program, Research Institute, Hospital for Sick Children, Toronto, Ontario, Canada. \\ "Department of Newborn and Developmental Paediatrics, Sunnybrook and Women's, Toronto, Ontario, Canada. \\ "Department of Pediatrics, University of Toronto, Ontario, Canada. \\ ** Toronto General Research Institute, Toronto General Hospital, Ontario, Canada. \\ ${ }^{\dagger t}$ Bloorview Research Institute, Bloorview Kids Rehab, Toronto, Ontario, Canada.
}

\begin{abstract}
Potentially significant numbers of infants hospitalized in Neonatal Intensive Care Units (NICUs) and Pediatric Intensive Care Units (PICUs) experience chronic pain. However, the phenomenon of chronic pain in infancy has neither been defined nor described adequately by researchers. To stimulate and focus further work in the area, the purpose of this study was to explore expertopinions on definitional and assessment parameters of infant chronic pain. Forty-five health care professionals, with a median of 17 years of clinical experience, were recruited from 4 tertiary-level, university-affiliated institutions. Individual $(n=24)$ and group $(n=21)$ interviews were conducted by trained interviewers. Qualitative data were analyzed using a standard descriptive method. Health care professionals were able to offer preliminary definitions of chronic pain in infants. The most contentious definitional issue was whether iatrogenically prolonged pain (pain induced and maintained by medical procedures) should be considered chronic pain. Possible indicators for chronic pain included inability to settle, social withdrawal, constant grimacing, tense body, hypo- or hyper-reactions to acute pain, and dysregulated sleep or feeding patterns. These indicators differed significantly from those traditionally used to measure acute pain.

Perspective: Despite infants' established capacity to physiologically experience chronic pain, no current definitions exist that are wholly applicable to infancy. By exploring the definitional parameters and potential assessment cues of infant chronic pain, this study provides a foundation for improving pain measurement and management in infants with chronic pain.
\end{abstract}

Key words: Chronic pain, infant, health professional.

recent consensus statement acknowledges that infants can experience chronic pain. ${ }^{3}$ However, there is no clear definition for chronic pain in infants, nor are there validated assessment scales. As a result, long-term pain may be inadequately managed in a vulnerable population. In older children and adults, chronic pain has been defined as pain without apparent biological value that has simply persisted or persisted beyond expected tissue healing time. ${ }^{2}$ Temporal delineations of "healing time" have been debated, ${ }^{6}$ as some suggest that pain could be considered chronic even if it lasts less than 1 month, ${ }^{15}$ whereas some offer definitions that stipulate pain should persist for 3 months ${ }^{8}$ or 6 months ${ }^{9}$ to be considered chronic.

Most of these timelines have the potential to systematically exclude the neonate and young infant simply 
because they have not lived long enough to fit these temporal criteria. Moreover, the concept of "normal healing time" in very young infants is poorly understood, especially in preterm neonates, in whom the gestational age of fetal viability continues to transition downward. Given the lack of a clear definition of chronic pain in infants and the considerable variability in how chronic pain is defined even in adults, it is not surprising that there is little research exploring potential assessment indicators for infant chronic pain.

A review of the literature produced 4 papers on pain beyond acute that could provide potential cues for infant chronic pain (Table 1). ${ }^{1,4,5,7,17}$ Although all of these researchers list potential indicators for "prolonged" or "persistent" pain in infants, there is a pronounced lack of agreement among them, particularly in the roles of physiological indicators, observation time periods, the role of cry, and type of facial expression. These inconsistencies are partly owing to the lack of consensus regarding when to use the terms "persistent," "prolonged," or "chronic" pain. Before one can properly assess and treat chronic pain in infants, a clearer articulation of a definition and key indicators for assessing chronic pain must be established., 74,16

Infants in Neonatal Intensive Care Units (NICUs) and Pediatric Intensive Care Units (PICUs) undergo many medical procedures that expose them to pain for long periods and are a population who may also suffer from chronically painful conditions (such as epidermolysis bullosa). Accordingly, clinical experts with these infants are an appropriate population to target in an exploratory study of how to best define chronic pain in infancy. Because prolonged pain is known to have both immediate and long-term consequences, it is crucial to understand this phenomenon more clearly. The contribution of experienced NICU clinicians is critical to the conceptualization of chronic pain in infancy. This descriptive exploratory study aims to elicit the opinions of highly experienced clinicians on the breadth of parameters that could be used to define and assess chronic pain in infancy.

\section{Materials and Methods}

\section{Study Population}

A primary selection recruitment strategy (researchers were aware of which specific individuals of a particular group would have the requisite knowledge to provide an informed response) was used to provide informationrich cases for in-depth study of this largely unexplored phenomenon (Morse, 1991). A purposeful sample (participants in our sample were selected for their level of clinical experience with infants) of 45 expert clinicians was recruited from 3 tertiary-level NICUs and 1 PICU in 3 university-affiliated hospitals in central Canada. Participants were recruited over a 10-month period spanning 2005 to 2006. Participants were considered clinical experts and were invited to participate if they (1) had a minimum of 10 years of professional experience caring for infants in an NICU or PICU setting (on 1 unit a lower boundary of 5 years was accepted as there were no clini- cians within a particular profession that met this criterion); (2) worked more than 19 hours per week; and (3) spoke English. An attempt was made to select as broad a sample as possible in terms of profession, educational preparation, and practice site. In total, the sample consisted of 26 nurses (58\%), 7 neonatologists (16\%), 7 respiratory therapists $(16 \%), 2$ pharmacists $(4 \%)$, and 1 of each from the following disciplines (6\% total): occupational therapy, physical therapy, and nutritional science. Preliminary data analysis occurred concomitantly with data collection, and saturation (when no new information is added to the data pool by subsequent participants) was used as the criterion to determine the end of recruitment. The median level of experience for the sample was approximately 17 years (Fig 1), $77 \%$ of the sample was female, and $85 \%$ were between 36 and 55 years of age ( $9 \%$ were younger and $6 \%$ were older).

\section{Procedure}

The study protocol was approved by research ethics boards at the participating hospitals and universities. Eligible participants were identified, using the inclusion criteria, by nurse managers (not otherwise involved in the study) on each of the participating units. All approached health care professionals agreed to participate and provided informed consent.

Participants were interviewed either individually or in groups. Two interview modalities (individual and group) were used to maximize the numbers of eligible staff that could participate during the study period. Twenty-one participants $(47 \%)$ were interviewed in a group format, whereas 24 participants (53\%) were interviewed individually. All participants answered every posed question, and no one declined to comment during any part of the interview. Interviews were conducted by the lead author and 2 of her doctoral-level clinical psychology graduate students (S.A. and L.D.). All interviews were audiotaped, transcribed verbatim, and then doublechecked for accuracy by a second transcriber.

\section{Semi-structured Interviews}

The structure of the interview guide was based on previously conducted work by the co-investigators ${ }^{10,11}$ to maximize idea generation from participants. Health care professionals were asked to describe an infant or a group of infants that they cared for that may fit into 1 of the following categories: (a) infants in pain for longer than expected given their medical status, (b) infants in pain for a longer period of time given what would be considered normal healing times for a particular procedure or procedures, and $(c)$ infants in pain that lasted for a long period of time. After they had provided a clinical example, participants were asked if they thought that the infant may have had "chronic pain" and to justify their rationale. This 2 -level sequencing of questions formed the basis for our analysis on opinions defining chronic pain. Respondents were also asked open-ended questions about (a) potential indicators of infant chronic pain and (b) potential medical conditions and situations that would characterize chronic pain in infants. 


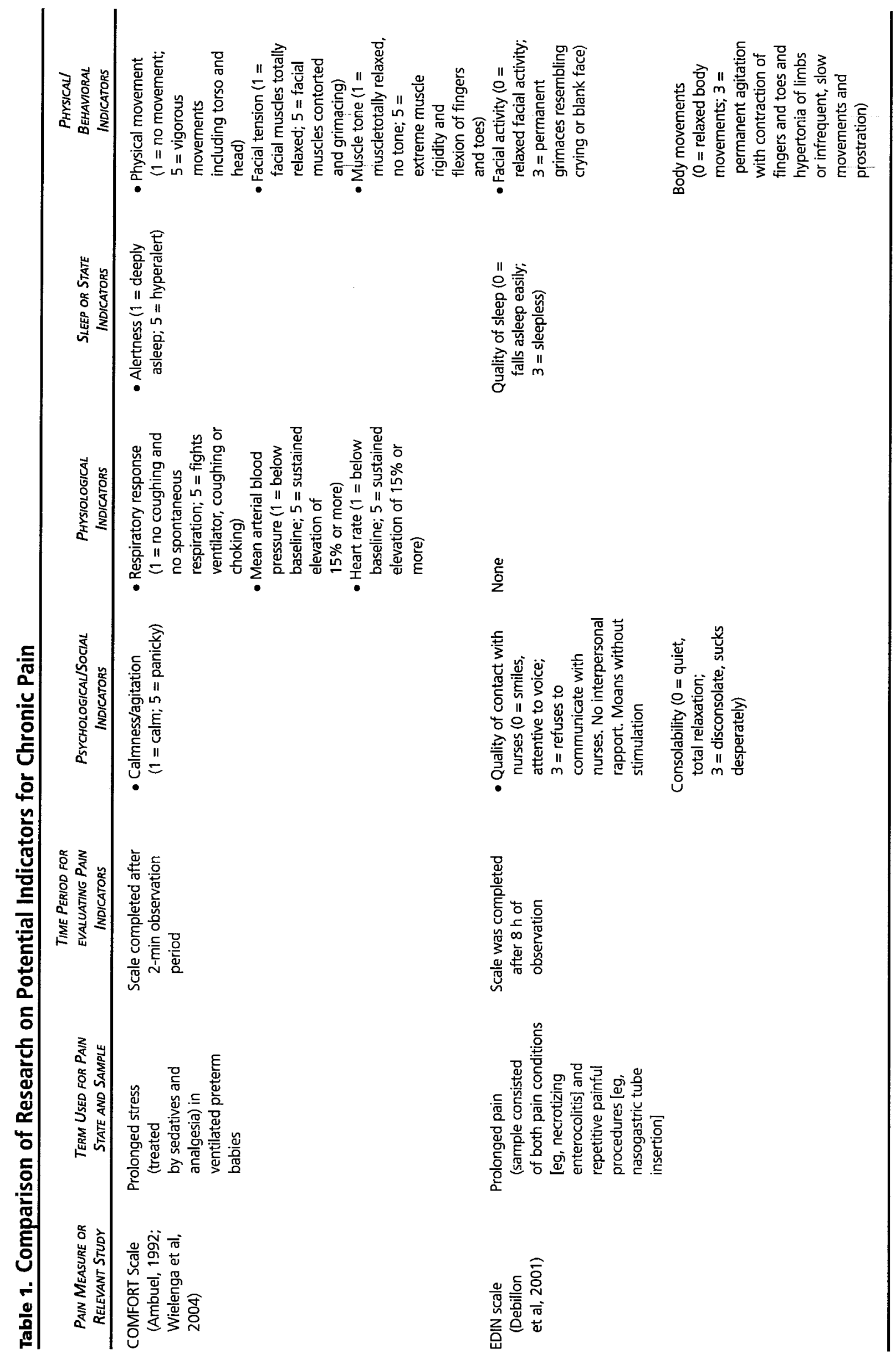




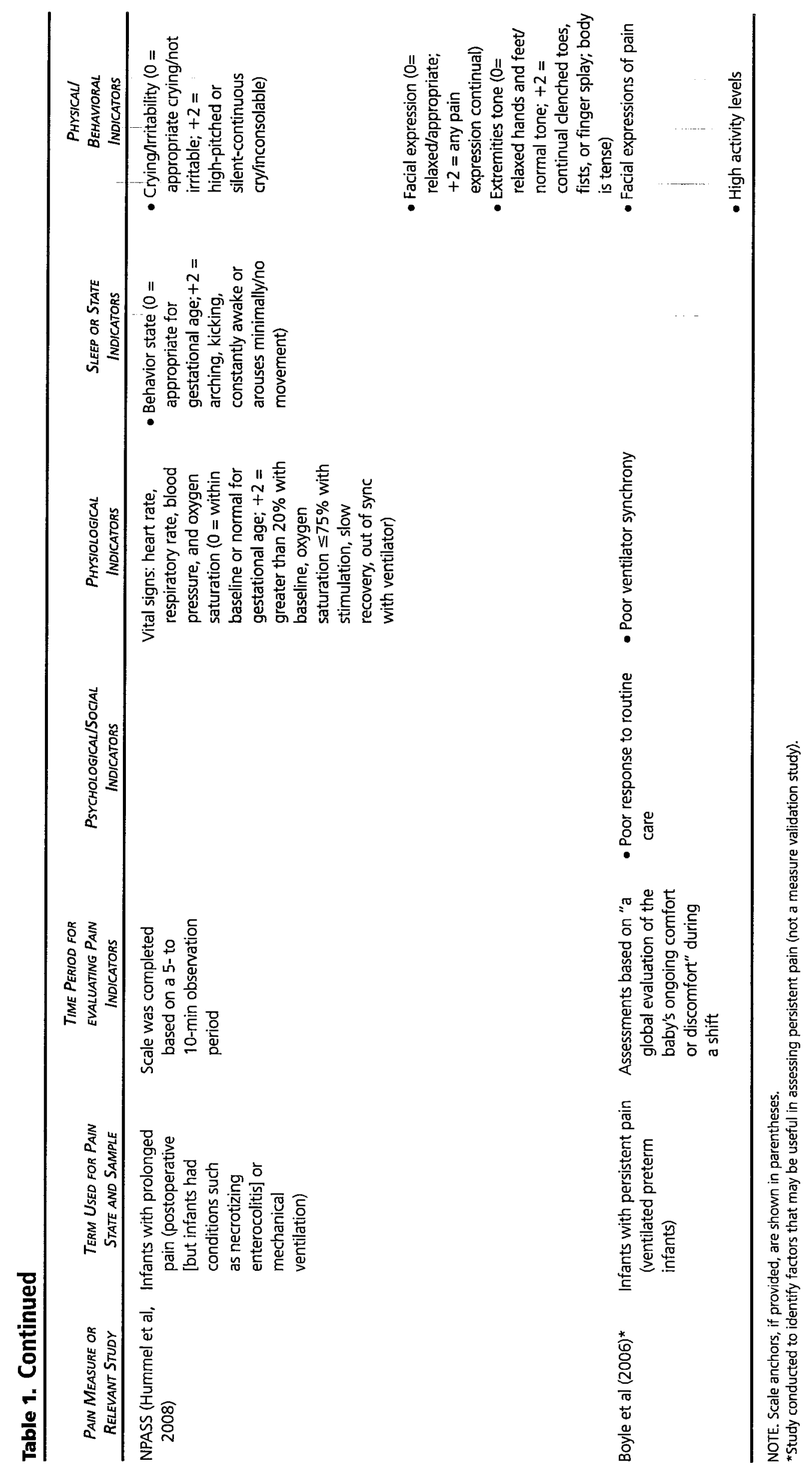




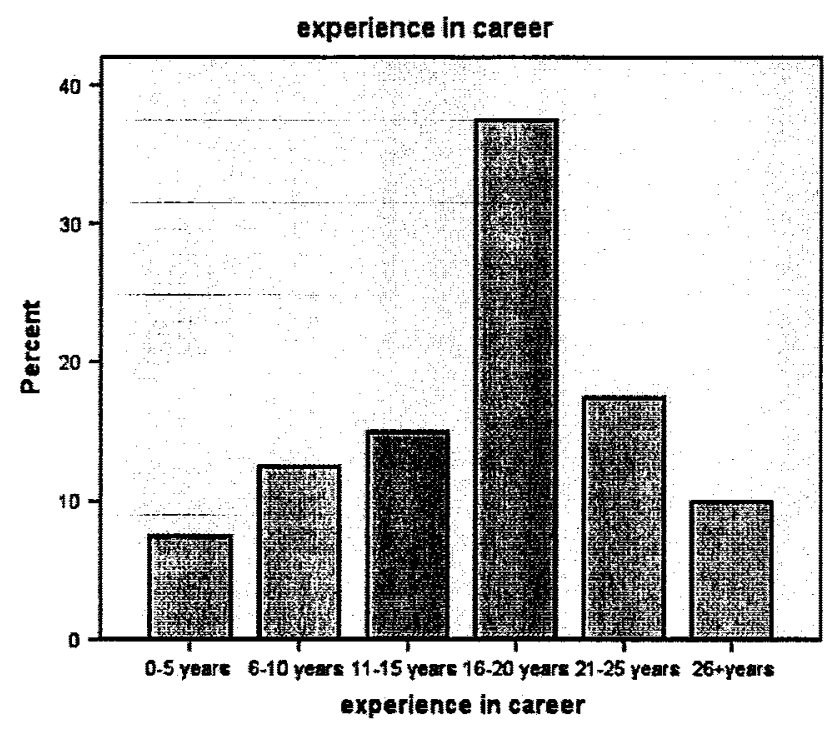

Figure 1. Years of experience of sample.

\section{Data Analysis}

Qualitative descriptive techniques explained by Sandelowski ${ }^{12,13}$ maximized the team's ability to organize ideas yet refrain from overinterpretation of the interview data. Qualitative description is a low-inference, categorizing approach that has the advantage of facilitating consensus by an analysis team and promotes a recapitulation of the objective observable "facts." Given that the primary purpose of this study was to generate a broad range of definitional and assessment parameters for chronic pain due to the lack of consensus in the field, subsidiary analyses (such as by responses by profession, site, interview modality) were seen as premature.

The analysis involved independent and group analysis procedures to describe the breadth of responses. The 28 transcripts (24 individual interview; 4 group interviews) were first distributed among 8 members of the team.
Every transcript was read and analyzed independently by 2 team members, 1 of which was always a PhD or MD member of the team. Using the interview schedule as a guide, team members were charged with listing every unique participant response under the 4 topic areas (infants capable of experiencing chronic pain, potential definitions of infant chronic pain, potential cues for infant chronic pain, potential examples of infant chronic pain). Next, a half-day group meeting, facilitated by the lead author, was held to bring the team together to obtain group consensus on what all the response categories for each of the 4 topic areas (infants can experience chronic pain, potential definitions of infant chronic pain, etc) were. All 8 team members participated in the half-day analysis meeting. After the exhaustive list of response categories had been agreed on by the whole team, each individual participant's transcript was quantitatively analyzed by a subgroup of the analysis team (R.P.R., L.D., and S.A.). Using NVivo 7.0, a frequency count was conducted using the agreed-on response categories as a framework. Any controversial transcript responses were discussed until group consensus was achieved.

Thus, if a participant mentioned a response category (in an open-ended interview, it is highly unlikely that a participant would ever give every response category within a topic area), they were classified as either agreeing, disagreeing, or being ambivalent to that response category (Tables 2 and 3). If a respondent gave a few potential ideas, they were counted in more than 1 response category for a given topic area. However, no participant was ever included twice within the same response category. Thus, for each response category within a topic area, 3 percentages are reported, reflecting the proportion of the total sample who agreed, disagreed, or were ambivalent. The topic areas pertaining to an infant's capability to experience chronic pain and potential examples of infant chronic pain were simply reported as percentages (see below).

Table 2. Response Categories for Topic Area: Potential Definitions for Infant Chronic Pain

\begin{tabular}{|c|c|c|c|c|}
\hline Response Categories & & AGREE & DISAGREE & AMBNALENT \\
\hline $\begin{array}{l}\text { Definition may be based on concept that chronic pain } \\
\text { in infants is extensive, repetitive exposure to acutely } \\
\text { painful procedures }\end{array}$ & $31 \%$ & & $31 \%$ & $2 \%$ \\
\hline Definition may be based on how long the infant is in pain & $49 \%$ & $\begin{array}{l}\text { Days: } 18 \% \\
\text { Weeks: } 20 \% \\
\text { Depends on diagnosis: } 7 \% \\
\text { Proportion of an infant's life: } 4 \%\end{array}$ & $14 \%$ & $0 \%$ \\
\hline $\begin{array}{l}\text { Definition may be based on the concept of pain lasting } \\
\text { longer than one would expect, given current } \\
\text { medical condition }\end{array}$ & $29 \%$ & & $4 \%$ & $0 \%$ \\
\hline $\begin{array}{l}\text { Definition may be based on concept that if it is } \\
\text { considered chronic pain in an adult or verbal child, it } \\
\text { is therefore chronic pain in an infant }\end{array}$ & $18 \%$ & & $0 \%$ & $0 \%$ \\
\hline $\begin{array}{l}\text { Definition may be based on concept that chronic pain } \\
\text { in infants is any pain that does not have a definite } \\
\text { end point }\end{array}$ & $22 \%$ & & $0 \%$ & $0 \%$ \\
\hline
\end{tabular}


Table 3. Response Categories for the Topic Area: Potential Cues for Infant Chronic Pain

Meta-Response Categories

- Cue Response Categories

AGREE DISAGREE AMBNALENT

1. Interacts with the environment/others

- Inability to settle/respond to comforting

- Lack of social interaction (not engaging with caregivers, avoiding eye contact)

- Will not respond to distraction-strategies

- Looking at infant's behavioral patterns over time to see differences from their baseline behavior

- Constant moaning, whimpering regardless of contact with caregivers

2. Face

- Face in reaction to pain is not as expressive; withdrawn expression

- Face has chronic grimacing, tension in the forehead

3. Body

- Issues with movement (grimacing on repositioning, resisting movement)

- Body appears tense (taunt legs and arms, clenched fists, scrunched toes)

4. Sleep patterns

- Restless sleep (not appearing restful when sleeping; not sleeping for long periods)

- Waking up startled

5. Physiological cues

- Not taking deep breaths, labored breathing

- Baseline elevation of heart rate

- Baseline elevation of cortisol (little change before and after painful procedure)

- Physiological signs are not useful

6. Reaction to acute pain

- Hyporeactivity: Not reacting when subjected to acute pain (no reaction, turning floppy after repeated pokes, appearing resigned)

- Hyperreactivity: Exaggerated acute pain response

- Does not respond to analgesics (still reacts to acute pain despite pain medication being given)

7. Feeding/bladder or bowel movements

- Problems with feeding (eg, not tolerating feeding, failure to gain weight)

- Problems with bladder and bowel (eg, not eliminating with regularity)

\begin{tabular}{|c|c|c|}
\hline $51 \%$ & 0 & 0 \\
\hline $31 \%$ & $2 \%$ & 0 \\
\hline $7 \%$ & $2 \%$ & 0 \\
\hline $27 \%$ & 0 & 0 \\
\hline $4 \%$ & 0 & 0 \\
\hline $20 \%$ & $2 \%$ & 0 \\
\hline $24 \%$ & 0 & 0 \\
\hline $27 \%$ & $2 \%$ & 0 \\
\hline $11 \%$ & $2 \%$ & 0 \\
\hline $18 \%$ & $2 \%$ & 0 \\
\hline $2 \%$ & 0 & 0 \\
\hline $4 \%$ & 0 & 0 \\
\hline $13 \%$ & $4 \%$ & 0 \\
\hline $4 \%$ & 0 & $4 \%$ \\
\hline $18 \%$ & $16 \%$ & $4 \%$ \\
\hline $53 \%$ & $4 \%$ & $2 \%$ \\
\hline $13 \%$ & $7 \%$ & $4 \%$ \\
\hline $7 \%$ & 0 & 0 \\
\hline $18 \%$ & 0 & 0 \\
\hline $2 \%$ & 0 & 0 \\
\hline
\end{tabular}

\section{Results}

The results are organized in relation to the major interview topic areas.

\section{Topic Area: Infants Are Capable of Experiencing Chronic Pain}

The vast majority of the sample gave an opinion and strongly agreed with the concept of infant chronic pain $(89 \%)$. Nine percent of the sample did not comment directly, $2 \%$ expressed ambivalence, and no one expressed disagreement. For example, 1 participant expressed the discomfort he/she felt about infants being in chronic pain but not being equipped to treat it properly.

"How dare we let our babies have chronic pain... I first thought that myself when I first heard you talk about the term chronic pain in infants... These children can't speak for themselves and so I should be treating them appropriately and how dare I let this happened...It's an uncomfortable topic." (Participant 1006)

\section{Topic Area: Potential Definitions for Chronic Pain in Infancy}

A broad range of opinions were offered when participants were asked about the parameters that define chronic pain. Five major response categories were discerned: duration of pain experience, pain that is longer than expected, any pain that is considered chronic in adult or older child populations, pain without a definite end point, and pain from repetitive painful procedures (Table 2; note rows do not tally to $100 \%$ because not every respondent gave every response [as would be expected]).

The most controversial response category ( $62 \%$ of sample gave an answer classified in this response category) revolved around whether chronic pain in hospitalized infants can be defined by repetitive acute procedures. Equal proportions of participants agreed and disagreed with this way of defining chronic pain. A few examples that typify the different perspectives include

\footnotetext{
"My definition of chronic pain is that it's not acute pain and if it's definitely not associated with a procedure or a surgery ...Procedural pain if it has a start and acute finish to an acute event....acute pain I see as there is a start, there is a finish. Chronic pain is ongoing." (Participant 1029)

"...so the baby has the tube in situ and it probably creates pain every single day but is it chronic because it is um you know, sort of, the pain, because IV is repeatedly moving in and out...this is not really chronic pain."(Participant 1030)

"I think it is pretty much the same (ie, chronic pain) if the infant that is getting repeated procedures that never recovers...versus exposing them to periodic procedural pain." (Participant 1034)
} 
"Accumulative pain from procedures-it's the same product as chronic pain." (Participant 1035)

Forty-nine percent of professionals also suggested that instead of looking at the event causing the pain, timelines may provide a potential way to define chronic pain. This response category had the largest amount of participant commentary. Of this subsample, $78 \%$ (38\% of total sample) thought days or weeks were the appropriate metric to define chronic pain in infancy.

Maybe we need a hybrid approach that when the pain is greater than our expectations, but our expectations for that baby with the excoriated peri-anal region will be in pain until the excoriation is healed , so in that situation you'd have to say anything longer than 24 hours... (Participant 1009)

Other potential suggestions, with $18 \%$ to $29 \%$ of the sample agreeing, related to defining chronic pain as pain beyond expectation/definite end point or just defining chronic pain in infancy as any pain type that would be considered chronic in adults.

"If there is a condition that causes pain and there is an expectation of how long the pain will last and the (infant's) pain lasts longer than the expectation... provided that the expectation is reasonable." (Participant 1019)

\section{Topic Area: Potential Examples of Chronic Pain in Infancy}

There were 5 major categories of responses for possible examples of chronic pain in infancy reflecting the diversity of responses offered for definitions. Four categories related to a chronic condition and 1 related to prolonged exposure to painful acute procedures. In descending order of response frequency, $33 \%$ gave an example of repetitive acutely painful procedures (eg, cardiac patients with multiple surgeries, infants exposed to daily heel lances, needle sticks, etc), $29 \%$ cited burns or skin conditions (eg, epidermolysis bullosa, necrotizing fasciitis), $28 \%$ gave a condition related to skeletal structure (eg, osteogenesis imperfecta, congenital contractures, osteopenia prematurity), and $22 \%$ selected a gastro-intestinal condition (eg, short gut syndrome, diaphragmatic hernia, reflux, necrotizing enterocolitis). Eighteen percent of the sample did not provide an example.

\section{Topic Area: Potential Cues for Chronic Pain}

Participants were asked about indicators (or cues) that might suggest that an infant is in chronic pain. To help prompt participants, participants were asked to offer cues for chronic pain that could be "behavioral, physiological, emotional, etc." Seven meta-response categories (categories of response categories; Table 3) were discerned: how the infant interacts with the environment/ others; facial expressions; body movements; sleep patterns; physiological cues; reaction to acute pain treat- ment; and cues relating to feeding, bladder, and/or bowel movements. The response categories under these meta-categories are listed in Table 3. Among the metacategories, 2 stood out because they contained at least 1 response category whereby at least $50 \%$ of the sample agreed on. The first was how the infant interacted with the environment/others (response categories included an inability to settle, a social withdrawal, and noting if the infant is acting "normal"). One example of a cue within this meta-category would be

"...they close down, don't want to look at you, and don't respond in the same way, we first have to determine if they have a neurological impairment or not...make sure you know that there is no other reason." (Participant 1006)

The second meta-category was the infant's reaction to acute pain; however, this indicator was controversial. Fifty-three percent of the sample was classified under the response category of a hyporeactivity to acute pain, whereas $20 \%$ were classified under the response category of a hyperreactivity to acute pain or evidence of a lack of response to analgesic medication. To exemplify the hyporeactivity response category, 1 participant noted

"It's a bit scary when you see it happens... you poke them they lie back, they have no movement, they don't cry anymore, they turn off, you see themthey almost, and you think "Oh they are not in pain anymore but they are." (Participant 1014)

To give an example of the hyperreactivity response category, another participant stated

"hypersensitive every time you approach them... react to acute pain in a way that is excessive." (Participant 1024)

The response categories clustered under the facial and body movement meta-categories tended to be prolonged physical cues that could be maintained over time (eg, chronic grimace, body looking tense). In the physiological cues meta-category, the most endorsed response suggested that physiological cues would not be good diagnostic cues for identifying chronic pain in infants. Finally, poor sleep (restless, waking up startled) or irregular elimination/feeding patterns were also offered as potential functional status indicators of chronic pain.

\section{Discussion}

\section{Definition of Chronic Pain in Infants}

There was a clear belief and general consensus that infants can experience chronic pain-a pain phenomenon that is distinct from acute procedural or postoperative pain. However, clinicians have differing notions of the term "chronic pain" and what the key constructs should be.

Findings from our pilot work ${ }^{10,11}$ on varying conceptualizations of chronic pain were validated. A definition that identified infant chronic pain as pain stemming from an unremitting painful condition (osteogenesis 
imperfecta, epidermolysis bullosa, short gut syndrome) or pain that lasted beyond expectation or without a definite end point was highly supported. However, defining chronic pain in infants by actual timelines or repetitive painful procedures ("iatrogenically prolonged") was more controversial. A large proportion of the sample, almost $40 \%$, suggested that infants who experienced pain for days or weeks (rather than month/months with verbal populations) may be experiencing chronic pain. The most controversial aspect of defining chronic pain in infants was whether the persistent pain from repetitive exposure to painful procedures (as is the reality of many infants hospitalized in the NICU or PICU) should also be considered as chronic pain. Some participants suggested that if pain was ongoing, regardless of whether the pain originated from an internal stimulus (ie, disease progression) versus an external stimulus (ie, repetitive surgeries, central line placements, etc), it should be considered chronic pain. Others objected from a treatment perspective, stating that pain with a definite end point (as in an iatrogenically prolonged pain state that resulted from repetitive acute procedures) should be handled differently than pain from a chronic condition such as epidermolysis bullosa. This debate emphasizes the urgent need to define pain states beyond acute phase in the NICU and PICU because without accurate assessment, proper treatment cannot ensue.

It should also be noted that these 2 sources of chronic pain are not mutually exclusive. Infants hospitalized in critical care units with an underlying painful disease progression are often exposed to painful acute procedures for prolonged periods of time. Therefore, we are hesitant to conclude from the present findings which definition is most representative of chronic pain in infants; however, it is clear that researchers and clinicians must make a concerted effort to come to some type of agreement.

\section{Indicators of Chronic Pain in Infants}

Despite a definitive definition being elusive, 7 groups of potential indicators for chronic pain were articulated.

However, 2 areas of response (regarding physiological cues and response to a painful acute stimulus) were noteworthy, as significant proportions agreed as disagreed with the utility of these areas. One possible explanation for this incongruity is that they may have different levels of usefulness in different stages of infant chronic pain. For example, infants with osteogenesis imperfecta may initially be hyperreactive to an acutely painful procedure but over time become hyporeactive when the pain continues (see below for more detailed discussion). A similar explanation may be applied to physiological indicators in that short-term physiological indicators may be useful but in the longer term, perhaps their utility decreases as the pain continues unabated.

Currently, as there is no clear way to distinguish between prolonged acute pain and chronic pain and no validated measures for assessing chronic pain in infants, measures in the area of prolonged pain provide a useful context in which to understand the current work. $4,5,7,17$ Our results are consistent with the existing literature sug- gesting that prolonged tense musculature (both facial and body) and agitated body movements could be key indicators for chronic pain. However, disagreement was noted on the importance of behavioral state and crying (in infants that are able to cry) in diagnosing pain beyond acute. Researchers who have helped to validate both the COMFORT $^{17}$ and the N-PASS ${ }^{7}$ measures include these 2 indicators, whereas these indicators do not appear in work by Debillion et $\mathrm{al}_{,}{ }^{5}$ Boyle et $\mathrm{al}^{4}{ }^{4}$ and the present study. Owing to our qualitative approach, we were able to glean more explanation on the importance of these factors from the caregivers. A low-grade intermittent moaning or whimpering was mentioned as more indicative of pain over long periods of time because many felt vigorous vocal responding [eg, "screaming or shrieking" (COMFORT); "high-pitched or silent continuous cry" (N-PASS)] would not be sustainable over a long period of time. Similarly, although the COMFORT and N-PASS measures have behavioral state as an indicator (with a "hyperalert" state being the high pain behavioral anchor), other researchers did not list these indicators as crucial. Although all measures were developed to assess pain beyond acute-procedural, the lack of a core group of consistent indicators typifies the current problem in the literature and seems to be reflective of the underlying lack of agreement on temporal delineations of prolonged acute versus chronic pain.

More inconsistencies were evident in relation to disorganized breathing (either labored breathing or fighting the ventilator). Although this area produced very minor mention by our participants and no mention in the EDIN, this indicator was included in the N-PASS and the COMFORT measures. However, tracking other vital signs (such as heart rate and blood pressure increases) was mentioned only by the N-PASS as a helpful strategy in assessing prolonged or chronic pain. Within our study, clinicians speculated that it was not sudden increases in physiologic indicators that could be potentially useful but rather a prolonged baseline elevation of indicators (ie, the consistent higher baseline heart rate because of ceiling effects on the parameters). Despite these specific assertions, it is noteworthy that the largest proportion of participants who opined on this indicator articulated that physiological indicators would not be useful in assessing chronic pain.

The EDIN appears to include cues that have a different focus than the other published prolonged/chronic pain measures for infants. Three of the indicators (quality of sleep, quality of interaction with nurses/caregivers, and consolability) are indicators that would not be assessed at 1 time point but rather reflect clinical judgments regarding a pattern of behavior over time. This focus on sleep and interactional patterns was also frequently mentioned by our sample of experienced health professionals. It appears logical that given the protracted nature of chronic pain, a key to assessing it as a distinct state from acute pain in nonverbal populations would involve an examination of patterns over time and/or the ability to distinguish these patterns as "different from usual" for a particular infant. Being able to distinguish "different from usual" behavioral patterns and responses 
suggests the importance of core caregiving for infants suffering from chronic pain.

Finally, there were unique indicators that emerged from our interviews that have not been documented in the literature to date. These indicators related to the intensity of the acute pain response and functional status (with the exception of sleep) of the infant. Two thirds of the sample articulated that an infant's reaction to a painful procedure would be key in assessing chronic pain. The vast majority of this subsample suggested that an infant that has little or no reaction to an acutely painful procedure may be experiencing chronic pain, whereas the remainder suggested it is an exaggerated response that is key. Again, this dichotomy may be due to the lack of consensus regarding timelines and potential confounding of other factors such as severity of illness and time in pain. For example, newborn infants may be initially highly reactive to an acute painful stimulus but after being in pain over time or having an increased severity of illness, the infant may run low on physical reserves to sustain a response; or the infant may learn that mounting a high response is not helpful and therefore stops or minimizes responding to conserve energy (ie, learned helplessness). In terms of changes to functional status, another indicator identified by a substantial proportion of the sample related to problems with feeding. Repeating the theme of examining the infant over time, a notable minority of the sample also suggested that examining how well an infant tolerates feeds and gains weight as potentially indicating chronic pain.

\section{References}

1. Ambul B, Hamlett KW, Marx CM, Blumer JL: Assessing distress in pediatric intensive care environments: The COMFORT Scale. J Pediatr Psychol 17:95-109, 1992

2. American Pain Society: Pediatric Chronic Pain, A Position Statement from the American Pain Society. Glenview, IL, American Pain Society, 2001

3. Anand KJS, Aranda JV, Berde CB, Buckman S, Capparelli EV, Carlo W, Hummel P, Johnston CC, Lantos J, Tutag-Lehr V, Lynn AM, Maxwell LG, Oberlander TF, Raju TNK, Soriano SG, Taddio A, Walco GA: Summary proceedings from the Neonatal Pain-Control Group. Pediatrics 117:S9-S22، 2006

4. Boyle E, Freer Y, Mae Wong C, McIntosh N, Anand K: Assessment of persistent pain or distress and adequacy of analgesia in preterm ventilated infants. Pain 124:87-91, 2006

5. Debillon T, Zupan V, Ravault N, Magny JF, Dehan M: Development and initial validation of the EDIN scale, a new tool for assessing prolonged pain in preterm infants. Arch Dis Child 85:F36-F40, 2001

6. Harstall C, Ospina M: How prevalent is chronic pain? Pain: Clinical Updates 11:1-4, 2003

7. Hummel P, Puchalski M, Creech SD, Weiss MG: Clinical reliability and validity of the N-PASS: Neonatal pain, agitation and sedation scale with prolonged pain. J Perinatol 28: $55-60,2008$

8. Jovey RD: Pain pathways and pathophysiology, in Jovey RD (ed): Managing Pain: The Canadian Healthcare
This is the first prospective examination of definitional and potential assessment cues for chronic pain in infancy from the perspective of highly experienced neonatal and infant clinicians. Chronic pain must be defined for infants, particularly in relation to pain that lasts for a protracted amount of time with a known end point (as is the case with iatrogenically prolonged pain in a NICU) and pain that occurs without an end point (such as infants with osteogenesis imperfecta). This definition must be sensitive to the specific physiological and behavioral development of an infant so that appropriate cues for chronic pain in infancy can be gleaned. This careful debate, scrutinized from a prospective vantage point, used the collective and extensive expertise of highly experienced infant health professionals. In doing so, it provides a strong foundation for measurement development that is not based simply on an extension of our infant acute pain knowledge base. Rather, new indicators for chronic pain in infants were generated and existing indicators were discussed. This work paves the way for a targeted observational study and chronic pain tool development in which behavioral, physical, functional, and interaction patterns over time may be most diagnostic.

\section{Acknowledgments}

We would like to acknowledge Dr Steven's Signy Hildur Eaton Chair in Paediatric Nursing Research Award at the Hospital for Sick Children and Dr Katz's Tier 1 Canada Research Chair in Health Psychology Award at York University.

Professional's Reference. Toronto, ON, Healthcare \& Financial Publishing, Rogers Media, 2002, pp 7-13

9. McAlpine L, McGrath PJ: Chronic and recurrent pain in children, in Block AR, Kremer EF, Fernandez E (eds). Handbook of Pain Syndromes. Mahwah, NJ, LEA. 1999, pp 529-549

10. Pillai Riddell RR, Stevens BJ: Invited response to Boyle, Freer, Wong, Mclntosh, and Anand on editorial "Looking Beyond Acute Pain in Infancy." Pain 127:302-304, 2007

11. Pillai Riddell R, Stevens BJ, Yamada J, Law M, Gibbins S, Asztalos E, McKeever P: Understanding of Chronic Pain in Infancy. Poster presented at the Annual Child Health Psychology Conference, Gainesville, FL, April 2006

12. Sandelowski M: "To be of use": Enhancing the utility of qualitative research. Nurs Outlook 45:125-132, 1997

13. Sandelowski M: Whatever happened to qualitative description? Res Nurs Health 23:334-340, 2000

14. Stevens BJ, Pillai Riddell RR: Looking beyond acute pain in infancy. Pain 124:11-12, 2006

15. Task Force on Taxonomy: Classification of Chronic Pain: Descriptions of Chronic Pain Syndromes and Definition of Pain Terms, (2nd edition). Seattle. WA, IASP Press, 1994 (reprinted 2002)

16. Whit-Hall R, Boyle E, Young T: Do ventilated neonates require pain management? Semin Perinatol 31:289-297, 2007

17. Wielenga JM, De Vos $R$, de Leeuw $R$, De Haan RJ: COMFORT scale: $A$ reliable and valid method to measure the amount of stress of ventilated preterm infants. J Neonatal Netw 23:39-44, 2004 